\title{
Effect of bovine lactoferrin as a novel therapeutic agent in a rat model of sepsis-induced acute lung injury
}

\author{
Nannan Han ${ }^{1}$, Hengjie Li ${ }^{1}$, Gang Li ${ }^{1}$, Ye Shen ${ }^{1}, \mathrm{Min} \mathrm{Fei}^{2}$ and Yong Nan ${ }^{1 *}$
}

\begin{abstract}
Sepsis is a serious clinical condition resulting from severe infection. High rates of mortality and tissue damage have been reported in intensive care unit (ICU) patients with sepsis. Bovine lactoferrin (BLF) is a well-known 80-kDa glycoprotein in the transferrin family that inhibits sepsis in low-birth-weight neonates. The present study investigated the protective effects of BLF in a rat model of sepsis-induced acute lung injury (ALI). The wet/dry ratio, lipid peroxidation, antioxidant markers, total protein, total cell count, inflammatory markers and myeloperoxidase (MPO) levels were assessed. Histopathological analysis was also carried out. BLF treatment reduced the wet/dry ratio of lung tissue by $30.7 \%$ and $61.3 \%$, and lipid peroxidation by $22.3 \%$ and $67 \%$, at concentrations of 100 and $200 \mathrm{mg} / \mathrm{kg}$, respectively. Superoxide dismutase (SOD), reduced glutathione (GSH), glutathione peroxidase (Gpx) and catalase were increased by more than $50 \%$ under treatment with $200 \mathrm{mg} / \mathrm{kg}$ BLF. Inflammatory markers, neutrophils, lymphocytes and total cell count were reduced by more than 50\% under treatment with $200 \mathrm{mg} / \mathrm{kg}$ BLF. BLF treatment significantly reduced MPO activity, by $28.2 \%$ and $74.3 \%$, at concentrations of 100 and $200 \mathrm{mg} / \mathrm{kg}$, respectively. Neutrophilic infiltration and edema were observed in control rats. However, BLF treatment restored intestinal microvilli to the normal range and reduced inflammatory cell invasion. Collectively, these results suggest that BLF is an effective therapeutic agent against sepsis-induced ALI.
\end{abstract}

Keywords: Bovine lactoferrin, Sepsis, Acute lung injury, Rats, Inflammation

\section{Introduction}

Sepsis is a serious clinical condition caused by severe infection (Taşc1 et al. 2017). Researchers have reported that sepsis is associated with high rates of mortality and tissue damage in intensive care unit (ICU) patients (Baracchi et al. 2011). Sepsis leads to multiple organ failure and lung dysfunction (Fujishima 2016). Acute lung injury (ALI) is associated with tachypnea and hypoxemia (Randhawa and Bellingan 2007), and researchers have reported that acute respiratory distress syndrome (ARDS) is linked with ALI (Matthay et al. 2012). ALI is associated with high rates of morbidity and mortality (Fang et al. 2012), and is responsible for 74,500 deaths

\footnotetext{
*Correspondence: nanyong8888@163.com

${ }^{1}$ Department of Emergency, Zhejiang Provincial People's Hospital/ People's Hospital of Hangzhou Medical College, Hangzhou 310014, Zhejiang, China

Full list of author information is available at the end of the article
}

per year in the Western countries (Walkey et al. 2012). Increased permeability of the alveolar-capillary membrane, pulmonary edema, accumulation of protein-rich fluid in the airspaces, poor lung performance, and pulmonary infiltration of neutrophils are key symptoms of ALI (Matute-Bello et al. 2008). Favarin et al. (2013) reported that there is currently no cure for ALI, and sepsis increases its mortality rate. Thus, identifying an effective therapeutic agent is a high priority.

Bovine lactoferrin (BLF) is a well-known $80-\mathrm{kDa}$ glycoprotein within the transferrin family. Manzoni et al. (2009) reported that BLF inhibits sepsis in low-birthweight neonates. Similarly, Chen et al. (2014) demonstrated the therapeutic effect of aerosolized BLF on lung injury and fibrosis in mice. Cutone et al. (2019) reported that aerosolized BLF reduced infection, iron imbalance and inflammation in chronic lung infection, and Hegazy et al. (2016) demonstrated renoprotective effects of BLF 
in acute kidney injury. Aerosolized BLF reduced proinflammatory cytokines and neutrophils in a mouse model of lung infection (Valenti et al. 2017). Therefore, the current study investigated the protective effect of BLF against sepsis-induced ALI in a rat model.

\section{Materials and methods}

Rats

Rats were obtained from the animal house of Zhejiang Province People's Hospital/People's Hospital of Hangzhou Medical College, China. The rats weighed 190-210 g and rats were kept in standard rat polypropylene cages $(435 \times 290 \times 150 \mathrm{~mm}$; six rats per cage $)$ and maintained under standard conditions of $12 \mathrm{~h}$ light $/ 12 \mathrm{~h}$ dark at a relative humidity of $60 \pm 5 \%$ and temperature of $25 \pm 0.5{ }^{\circ} \mathrm{C}$ with food and water provided ad libitum. All rats were maintained under appropriate conditions according to ethical standards for animal welfare.

\section{ALI and rat groups}

Experimental ALI was induced according to Filgueiras et al. (2012). Rats were divided into sham, control (ALI), ALI $+100 \mathrm{mg} / \mathrm{kg}$ body weight (bwt) BLF (L9507, SigmaAldrich, Shanghai, China), and $200 \mathrm{mg} / \mathrm{kg}$ bwt BLF groups. $24 \mathrm{~h}$ after ALI induction, rats were given BLF for 30 consecutive days through oral gavage. Sham and control rats were administered an equal volume of saline. At the end of the treatment, the blood and bronchoalveolar lavage fluid (BALF) were collected.

\section{Measurement of wet/dry ratio, lipid peroxidation and antioxidant markers}

The wet/dry ratio of lung tissue by weight was determined according to Huang et al. (2017). The levels of lipid peroxidation in fresh lung tissue homogenates were determined according to Toufekoula et al. (2013). Serum levels of superoxide dismutase (SOD), reduced glutathione (GSH), glutathione peroxidase (Gpx) and catalase were determined according to Zhang et al. (2019).

\section{Determination of protein content and total cell count}

In the BALF, the level of total protein was determined using the bicinchoninic acid (BCA) method according to Yalamati et al. (2015). Neutrophils, lymphocytes and total cells in the BALF were measured according to DomagałaKulawik et al. (2012).

\section{Determination of inflammatory markers and myeloperoxidase}

Migration inhibitory factor (MIF), interleukin-8 (IL-8) and tumor necrosis factor-alpha (TNF- $\alpha$ ) levels were measured using ELISA assay kits according to a previously reported method (Kothari et al. 2013). Levels of myeloperoxidase (MPO) in the homogenate were measured according to a previously reported method (Queiroz-Junior et al. 2009).

\section{Histopathological study}

Lung histopathological analysis was carried out according to a previously reported method (Althnaian et al. 2013). Briefly, the lower pulmonary lobes were immersed in $10 \%$ formalin and then embedded in paraffin. Then, 4- $\mu \mathrm{m}$-thick sections were prepared and stained by hematoxylin and eosin (H\&E). Lung tissue sections were examined under a light microscope.

\section{Statistical analysis}

Experimental results are given as mean \pm standard deviation (SD). All data were analyzed and compared using analysis of variance followed by Tukey's post hoc test. A threshold of $P<0.05$ was taken as significant.

\section{Results}

\section{Effect of BLF on wet/dry ratio of lung tissue}

We observed protective effects of BLF against sepsisinduced ALI in the rat model. The wet/dry ratio of tissue increased by $406.2 \%$ in the control rats (Fig. $1, P<0.05$ ). However, BLF treatment decreased the wet/dry ratio of lung tissue by $30.7 \%$ and $61.3 \%$ at concentrations of 100 and $200 \mathrm{mg} / \mathrm{kg}$, respectively (Fig. $1, P<0.05$ ).

\section{Effect of BLF on lipid peroxidation}

Lipid peroxidation was determined based on malondialdehyde (MDA) levels in the lung tissue homogenate. Lipid peroxidation dramatically increased, by $323.5 \%$, in the control rats compared to the sham rats. However, BLF treatment reduced the MDA content by $22.3 \%$ and $67 \%$ at concentrations of 100 and $200 \mathrm{mg} / \mathrm{kg}$, respectively (Fig. 2, $P<0.05$ ).

\section{Effect of BLF on antioxidants}

SOD, Gpx and catalase activity substantially decreased in the control rats. However, BLF treatment significantly increased SOD, catalase and Gpx activity, by over $50 \%$, at a concentration of $200 \mathrm{mg} / \mathrm{kg}$ (Fig. $3 \mathrm{a}-\mathrm{c}, P<0.05$ ). The GSH level decreased by $69.7 \%$ in the control rats. BLF treatment increased the GSH content by $69.6 \%$ and $169.5 \%$ at concentrations of 100 and $200 \mathrm{mg} / \mathrm{kg}$, respectively (Fig. 3d, $P<0.05$ ).

\section{Effect of BLF on total protein and total cells}

Total protein content increased by $381.3 \%$ in control rats compared to sham rats. BLF treatment reduced the total protein content by $27.2 \%$ and $68.7 \%$ at concentrations of 100 and $200 \mathrm{mg} / \mathrm{kg}$, respectively (Fig. 4, $P<0.05$ ). Lymphocytes, neutrophils and the total cell count increased 


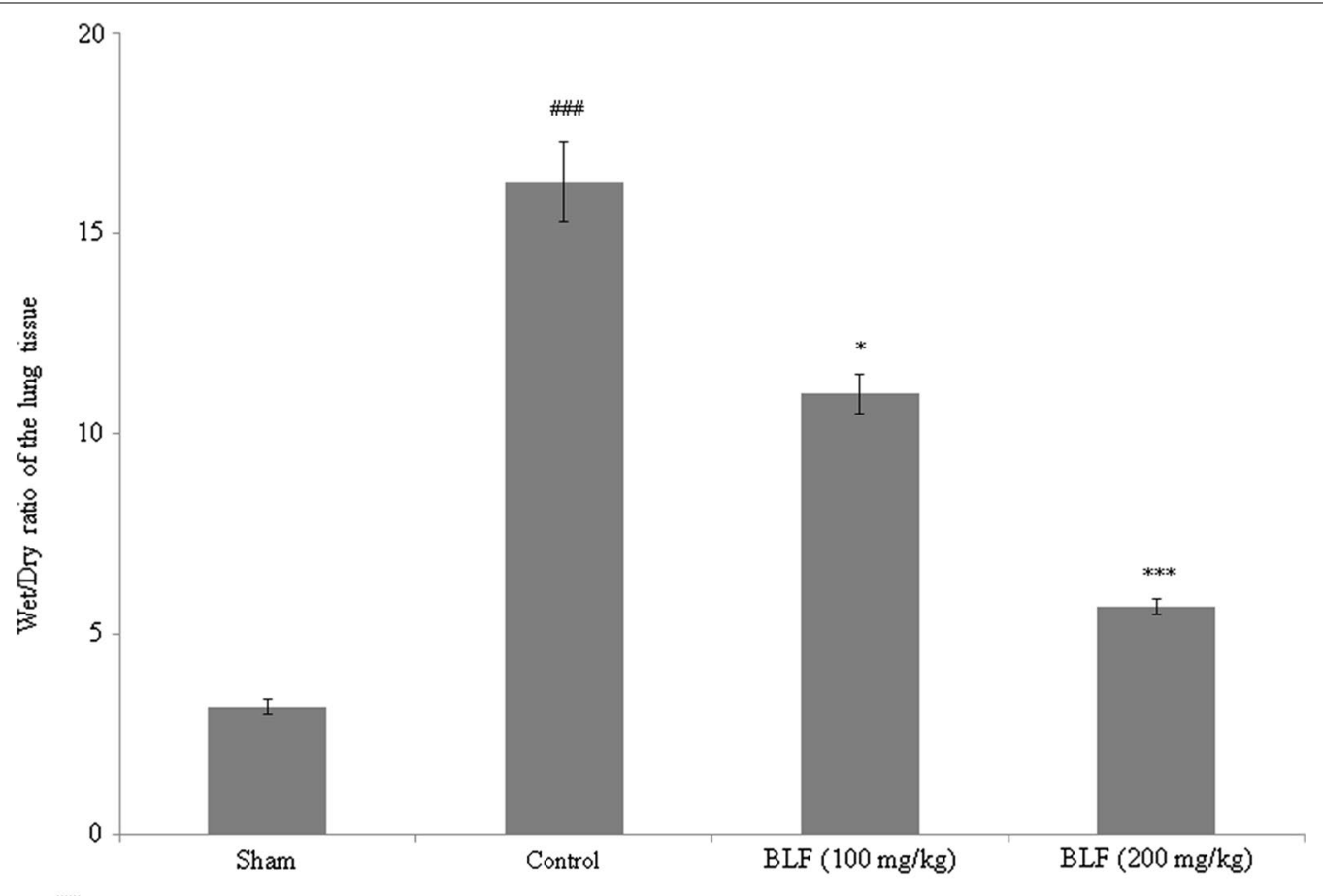

Fig. 1 Bovine lactoferrin (BLF) reduces the wet/dry ratio of lung tissue in acute lung injury (ALI)-induced male albino rats. Rats were given BLF for 30 consecutive days through oral gavage. ${ }^{\# \#} P<0.001$ compared to the sham group; ${ }^{*} P<0.05$ and ${ }^{* *} P<0.001$ compared to control rats

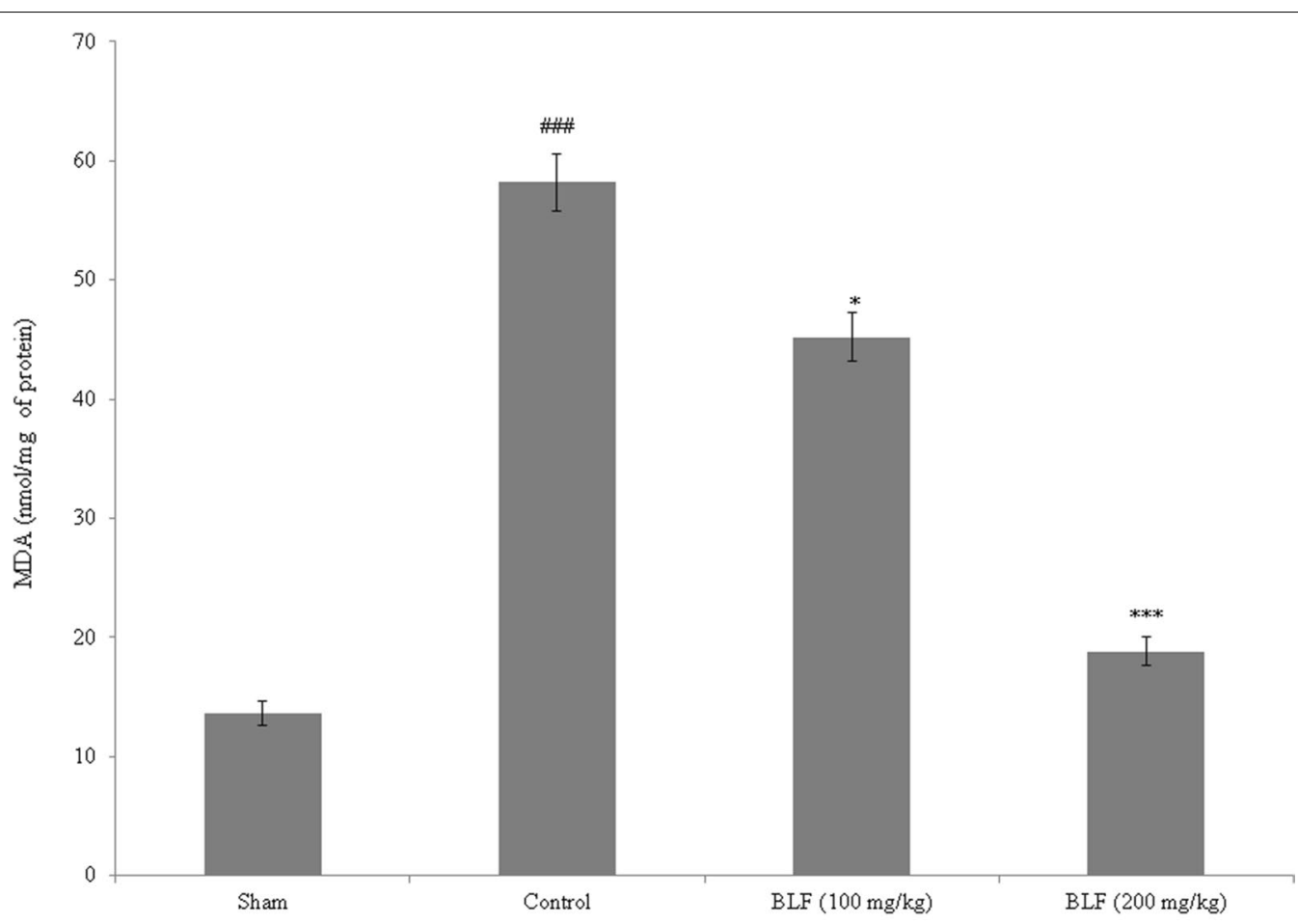

$P<0.001, * P<0.05 \& * P<0.001$

Fig. 2 BLF reduces lipid peroxidation in lung tissue homogenate of ALI-induced male albino rats. Rats were given BLF for 30 consecutive days through oral gavage. ${ }^{\# \#} P<0.001$ compared to the sham group; ${ }^{*} P<0.05$ and ${ }^{* * *} P<0.001$ compared to control rats 

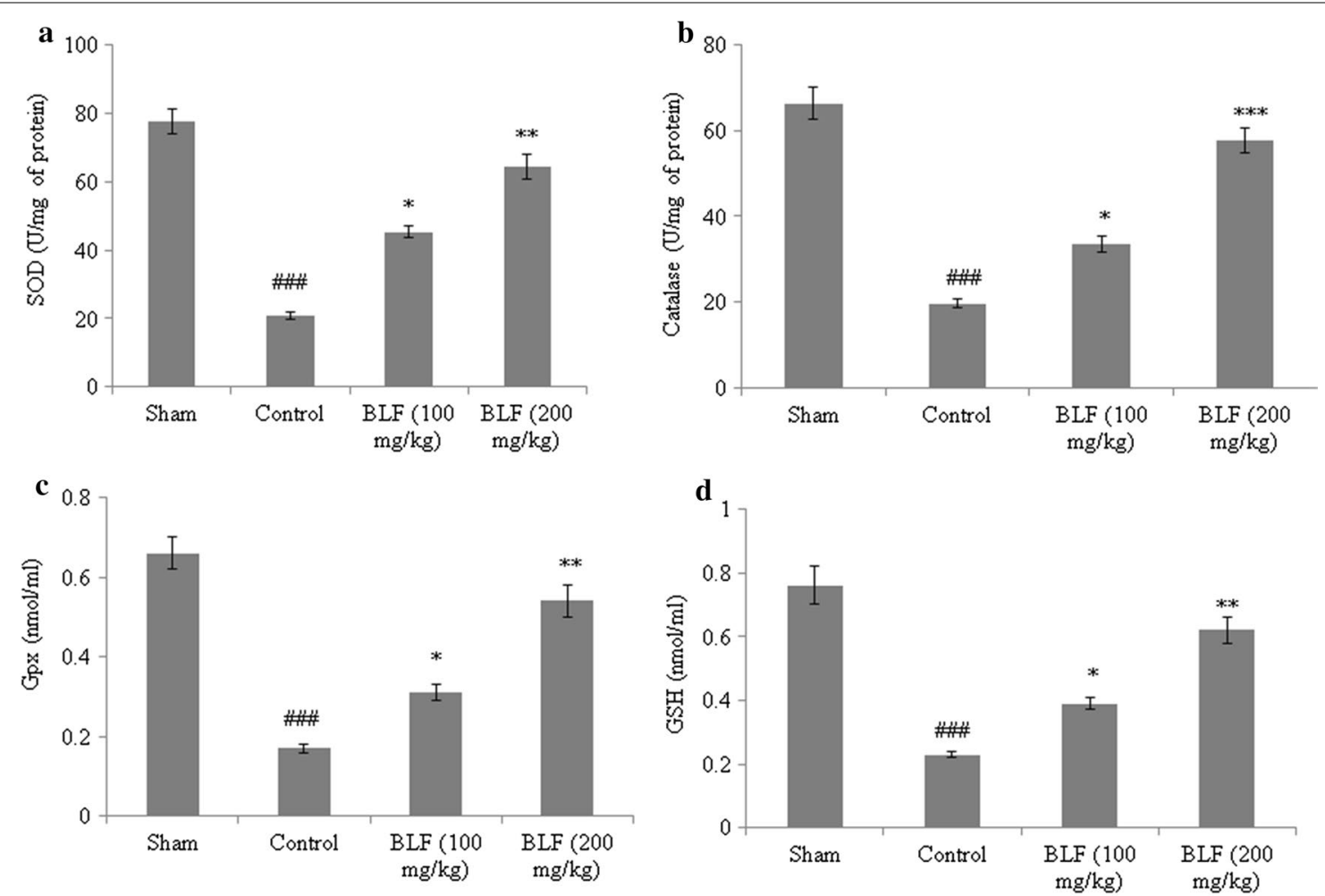

$P<0.001, * P<0.05, * * P<0.01 \& * * * P<0.001$

Fig. 3 BLF increases superoxide dismutase (SOD) (a), catalase (b), glutathione peroxidase (Gpx) (c) and glutathione (GSH) (d) in the lung tissue homogenate of ALI-induced male albino rats. Rats were given BLF for 30 consecutive days through oral gavage. \#\# $P<0.001$ compared to the sham group; ${ }^{*} P<0.05$ and ${ }^{* *} P<0.001$ compared to control rats

substantially in control rats, but were reduced by more than $50 \%$ under BLF treatment at a concentration of $200 \mathrm{mg} / \mathrm{kg}$ (Fig. $5 \mathrm{a}-\mathrm{c}, P<0.05$ ).

\section{Effect of BLF on inflammatory markers and myeloperoxidase}

The TNF- $\alpha$, IL- 8 and MIF levels increased in control rats, but decreased by more than $50 \%$ under BLF treatment at a concentration of $200 \mathrm{mg} / \mathrm{kg}$ (Fig. $6 \mathrm{a}-\mathrm{c}, P<0.05$ ). MPO activity was elevated by $588.6 \%$ in control rats relative to sham rats, but decreased by $28.2 \%$ and $74.3 \%$ under BLF treatment at concentrations of 100 and $200 \mathrm{mg} / \mathrm{kg}$, respectively (Fig. $7, P<0.05$ ).

\section{Effect of BLF on cellular architecture of lung tissue}

Histopathological analysis of the sham rats showed normal cellular architecture. Neutrophilic infiltration and edema were found in the control rats (Fig. 8). However, BLF treatment restored intestinal microvilli injury to the normal range and reduced inflammatory cell invasion (Fig. 8).

\section{Discussion}

We identified protective effects of BLF against sepsisinduced ALI in a rat model. Sepsis may lead to multiple organ failure and lung dysfunction (Fujishima 2016). Patients with ALI often present with tachypnea and hypoxemia (Randhawa and Bellingan 2007). ALI is associated with high rates of morbidity and mortality (Fang et al. 2012), and causes 74,500 deaths per year in Western countries Walkey et al. (2012). Increased permeability of the alveolar-capillary membrane, pulmonary edema, accumulation of protein-rich fluid in the airspaces, poor lung performance, and pulmonary infiltration of neutrophils are key symptoms of ALI (Matute-Bello et al. 2008). Favarin et al. (2013) reported that there is currently no cure for ALI, and that sepsis increases its mortality rate.

The destruction of the pulmonary endothelium and alveolar epithelium leads to lung dysfunction, which can result in complete failure of several organs in sepsis patients Ware and Matthay (2000). ILs, TNF- $\alpha$, and several inflammatory cytokines and mediators, are produced from activated macrophages and neutrophils (Chen et al. 2017). In our study, neutrophils, lymphocytes, total cell count, IL- 8 , TNF- $\alpha$ and MIF substantially increased in 


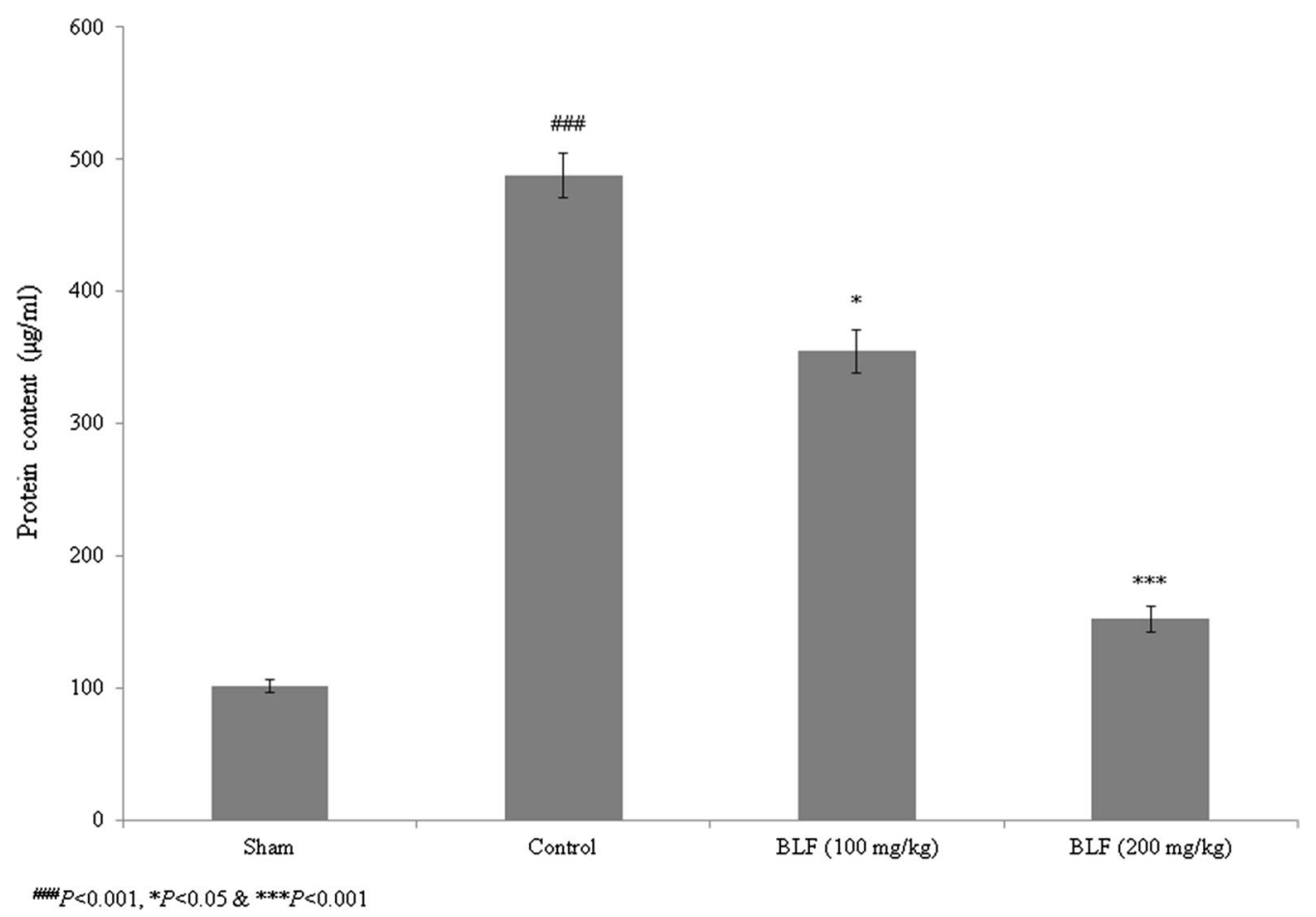

Fig. 4 BLF reduces total protein content in the lung tissue homogenate of ALI-induced male albino rats. Rats were given BLF for 30 consecutive days through oral gavage. ${ }^{\# \#} P<0.001$ compared to the sham group; ${ }^{*} P<0.05$ and ${ }^{* * *} P<0.001$ compared to control rats
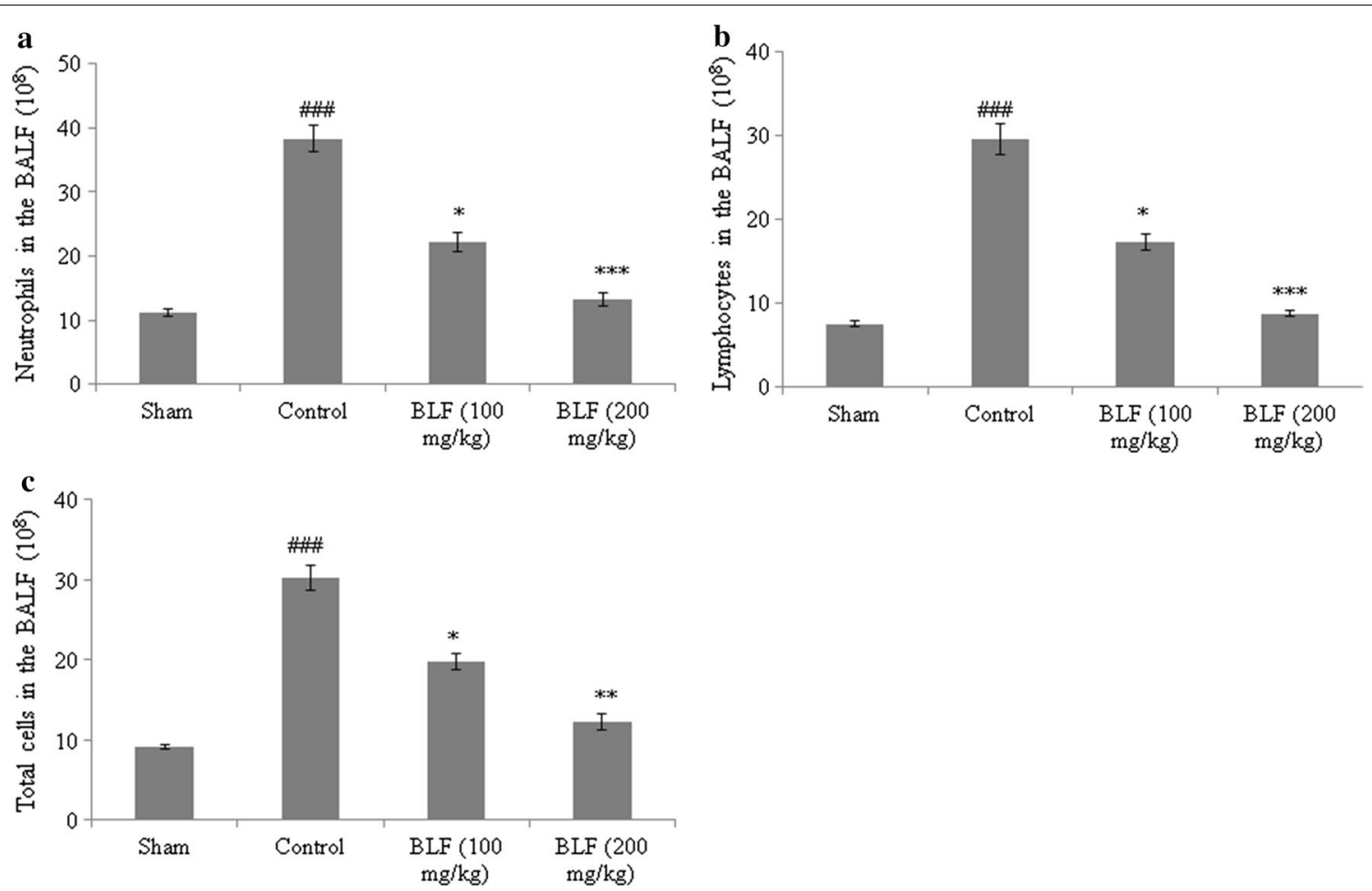

N $P<0.001, * P<0.05, * * P<0.01 \& * * * P<0.001$

Fig. 5 BLF reduces neutrophils (a), lymphocytes $(\mathbf{b})$ and total cell count (c) in ALI-induced male albino rats. Rats were given BLF for 30 consecutive days through oral gavage. ${ }^{\# \#} P<0.001$ compared to the sham group; ${ }^{*} P<0.05$ and ${ }^{* *} P<0.001$ compared to control rats 

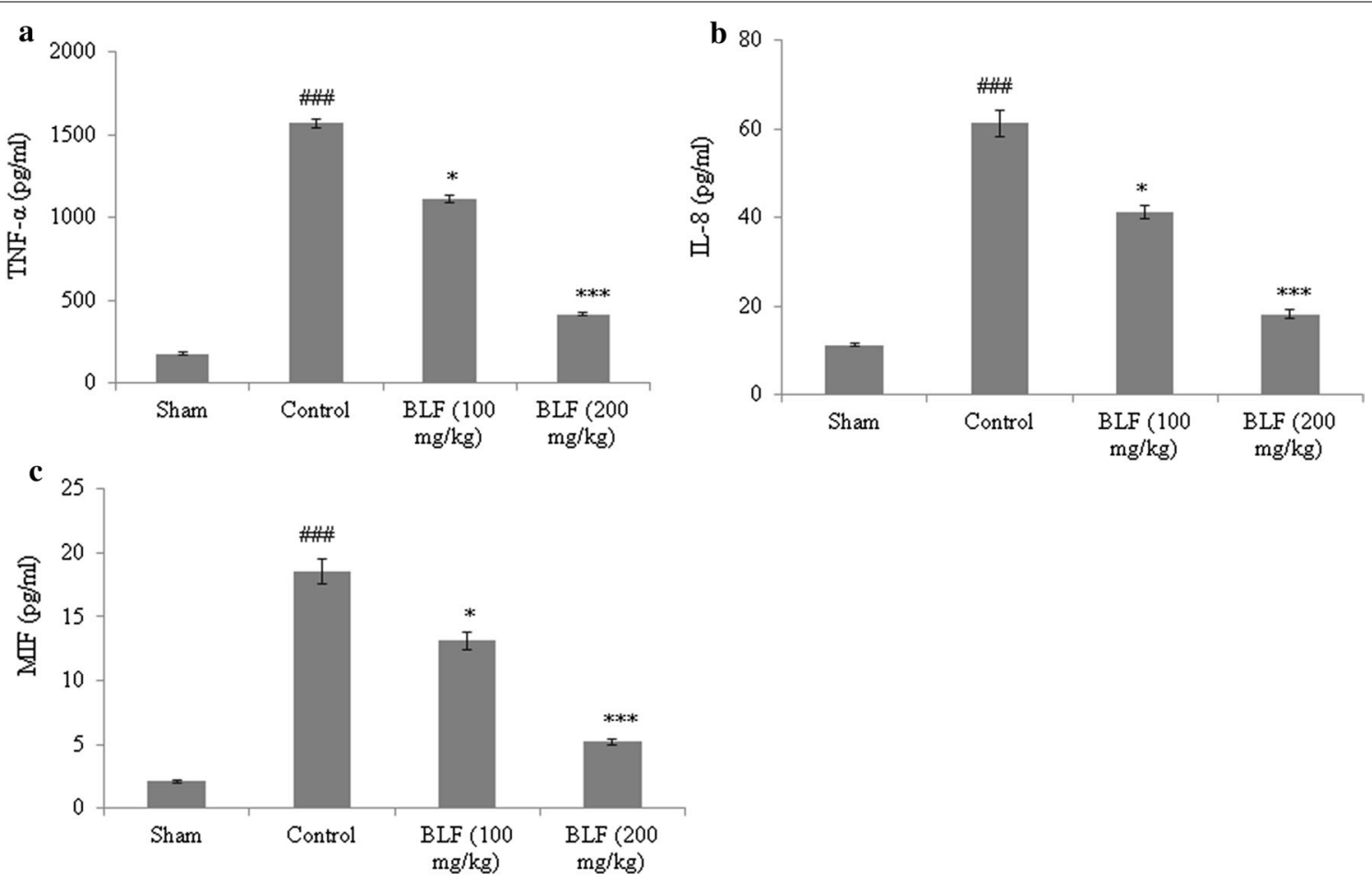

*\# $P<0.001, * P<0.05 \& * * * P<0.001$

Fig. 6 BLF reduces tumor necrosis factor-alpha (TNF-a) (a), interleukin-8 (IL-8) (b) and migration inhibitory factor (MIF) (c) levels in ALI-induced male albino rats. Rats were given BLF for 30 consecutive days through oral gavage. ${ }^{\# \#} P<0.001$ compared to the sham group; ${ }^{*} P<0.05$ and ${ }^{* * *} P<0.001$ compared to control rats

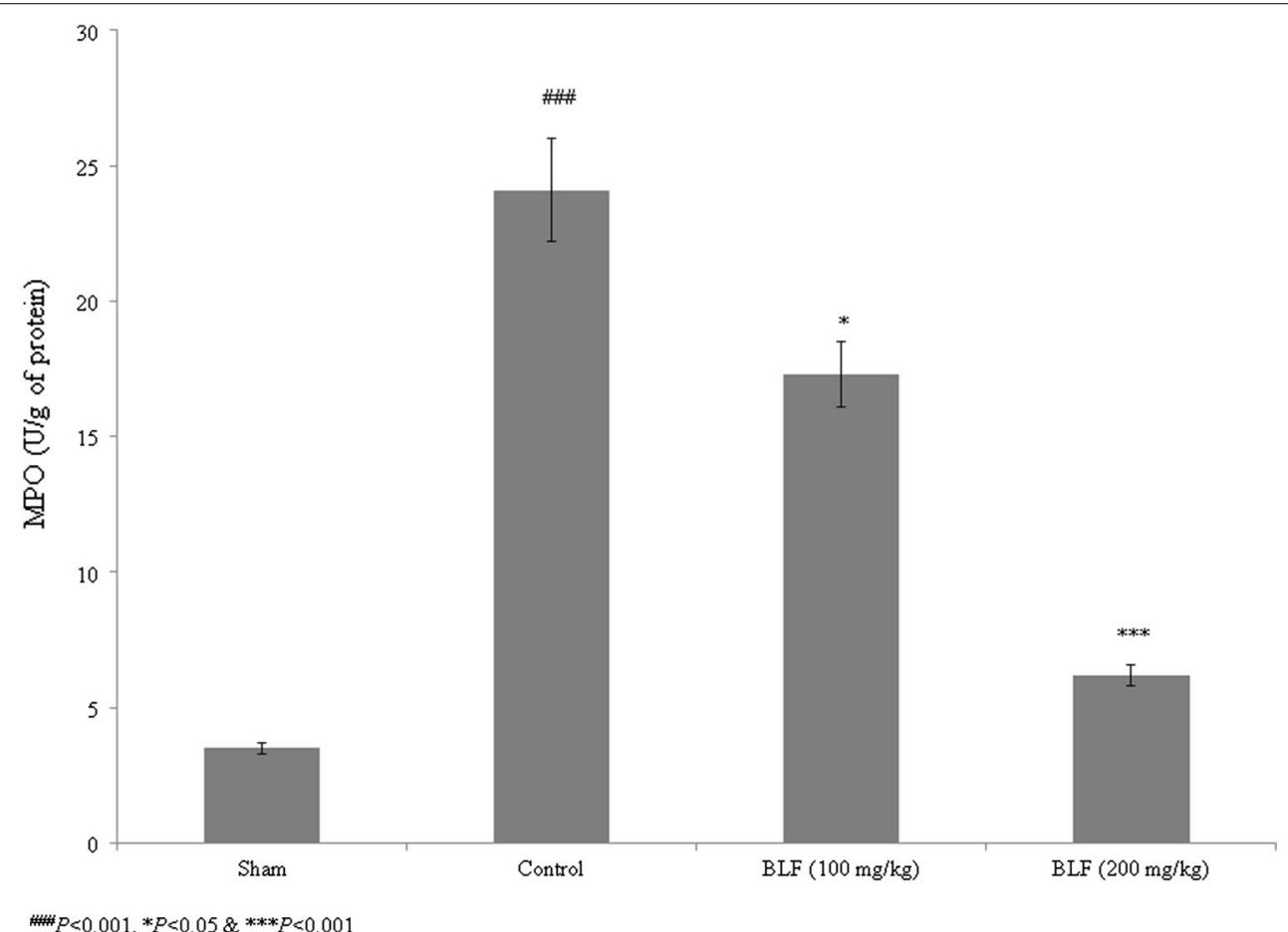

Fig. 7 BLF reduces myeloperoxidase (MPO) activity in ALI-induced male albino rats. Rats were given BLF for 30 consecutive days through oral gavage. ${ }^{\# \# \#} P<0.001$ compared to the sham group; ${ }^{*} P<0.05$ and ${ }^{* * *} P<0.001$ compared to control rats 

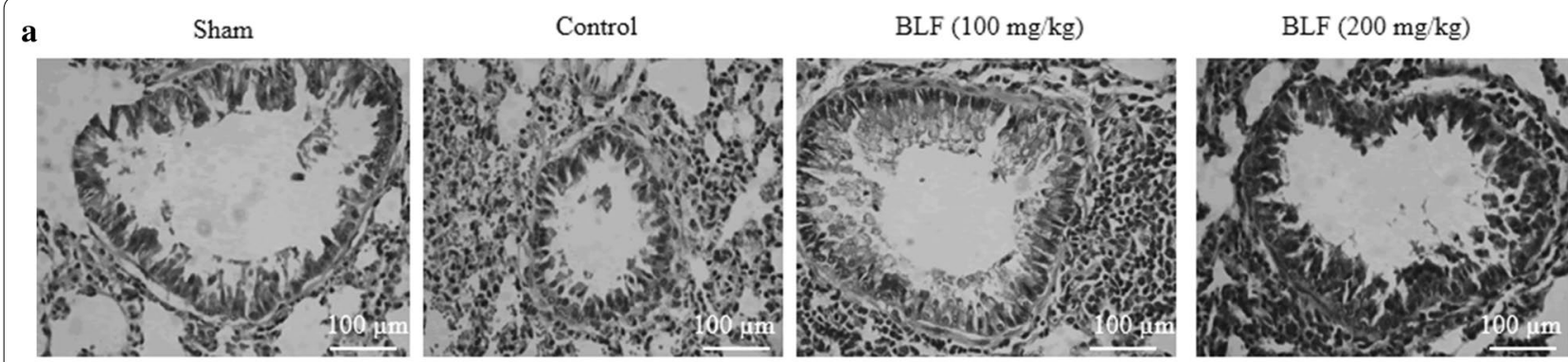

b

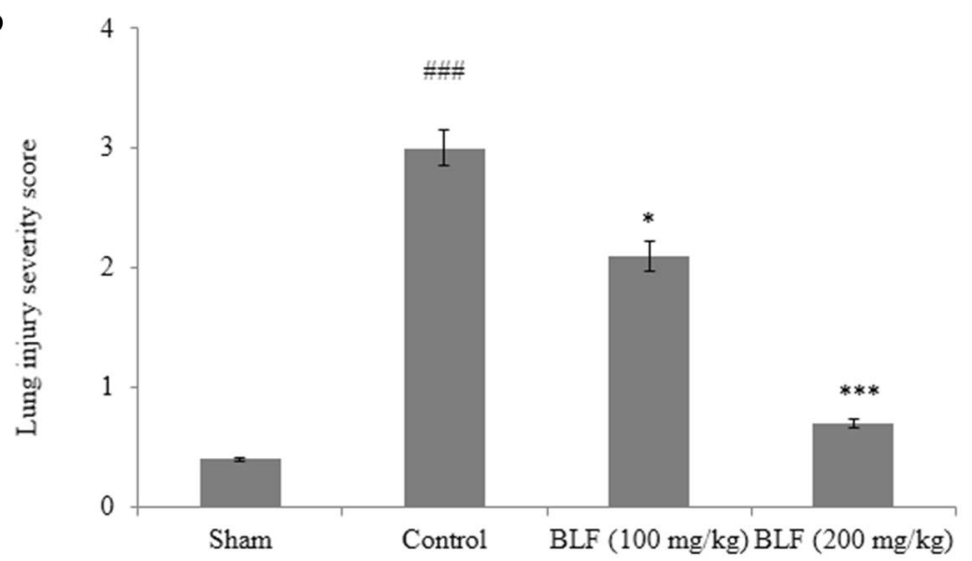

$=P<0.001, * P<0.05 \& * * * P<0.001$

Fig. 8 BLF restores intestinal microvilli damage to within the normal range and reduced inflammatory cell invasion in ALI-induced male albino rats. Rats were given BLF for 30 consecutive days through oral gavage. a Represents the histopathological images of lung tissues and $\mathbf{b}$ represents the lung injury severity score. ${ }^{\# \#} P<0.001$ compared to the sham group; ${ }^{*} P<0.05$ and ${ }^{* *} P<0.001$ compared to control rats. Scale bar is $100 \mu \mathrm{m}$

control rats (ALI). However, 100 and $200 \mathrm{mg} / \mathrm{kg}$ of BLF treatment reduced these markers to within the normal range. Manzoni et al. (2009) reported that BLF prevents sepsis in low-birth-weight neonates, and Chen et al. (2014) demonstrated a therapeutic effect of aerosolized BLF against lung injury and fibrosis in mice. Cutone et al. (2019) reported that aerosolized BLF reduced infection, iron dysbalance and inflammation in chronic lung infection. In a rat model of acute kidney injury, Hegazy et al. (2016) identified renoprotective effects of BLF. Valenti et al. (2017) showed that aerosolized BLF reduces proinflammatory cytokines and neutrophils in a mouse model of lung infection.

The pathological importance of oxidative stress in ALI has been highlighted by previous studies (Kellner et al. 2017). Reactive oxygen species (ROS) react with macromolecules, leading to higher rates of lipid peroxidation (Kwiecien et al. 2014). Bhattacharyya et al. (2004) reported that neutrophils produce superoxide molecules during inflammation. In the present study, lipid peroxidation substantially increased in control rats (ALI). However, 100 and $200 \mathrm{mg} / \mathrm{kg}$ of BLF treatment reduced lipid peroxidation. This is consistent with the results of Faridvand et al. (2017) who showed that BLF treatment inhibited lipid peroxidation in rats. Collectively, these results suggest that BLF is an effective therapeutic agent against sepsis-induced ALI.

\section{Acknowledgements \\ None.}

Authors' contributions

$\mathrm{NH}, \mathrm{HL}$ and $\mathrm{GL}$ conducted experiments and collected data. YS, MF and YN carried out data interpretation, review of literature and manuscript drafting. All authors read and approved the final manuscript.

\section{Funding}

This project is funded by the Zhejiang Provincial Chinese Medicine Technology Project (No. 2017ZA007)

\section{Availability of data and materials}

Corresponding author could provide the all experimental data on valid request.

\section{Ethics approval and consent to participate}

All the animal experiments were approved by ethics committee of Zhejiang Province People's Hospital/People's Hospital of Hangzhou Medical College, Hangzhou, Zhejiang Province, 310014, China.

\section{Consent for publication}

Not applicable. 


\section{Competing interests}

The authors declare that they have no competing interests.

\section{Author details}

${ }^{1}$ Department of Emergency, Zhejiang Provincial People's Hospital/People's Hospital of Hangzhou Medical College, Hangzhou 310014, Zhejiang, China. ${ }^{2}$ Health Examination Centre, Zhejiang Provincial People's Hospital/People's Hospital of Hangzhou Medical College, Hangzhou 310014, Zhejiang, China.

Received: 21 August 2019 Accepted: 17 October 2019

Published online: 31 October 2019

\section{References}

Althnaian T, Albokhadaim I, El-Bahr SM (2013) Biochemical and histopathological study in rats intoxicated with carbontetrachloride and treated with camel milk. Springerplus 2(1):57

Baracchi F, Ingiosi AM, Raymond RM, Opp MR (2011) Sepsis-induced alterations in sleep of rats. Am J Physiol Regul Integr Comp Physiol 301:R1467-R1478

Bhattacharyya J, Biswas S, Datta AG (2004) Mode of action of endotoxin: role of free radicals and antioxidants. Curr Med Chem 11:359-368

Chen HL, Yen CC, Wang SM, Tsai TC, Lai ZL, Sun JY, Lin W, Hsu WH, Chen CM (2014) Aerosolized bovine lactoferrin reduces lung injury and fibrosis in mice exposed to hyperoxia. Biometals 27(5):1057-1068

Chen L, Deng H, Cui H, Fang J, Zuo Z, Deng J, Li Y, Wang X, Zhao L (2017) Inflammatory responses and inflammation-associated diseases in organs. Oncotarget 9:7204-7218

Cutone A, Lepanto MS, Rosa L, Scotti MJ, Rossi A, Ranucci S, De Fino I, Bragonzi A, Valenti P, Musci G, Berlutti F (2019) Aerosolized bovine lactoferrin counteracts infection, inflammation and iron dysbalance in a cystic fibrosis mouse model of Pseudomonas aeruginosa chronic lung infection. Int J Mol Sci 20(9):2128

Domagała-Kulawik J, Skirecki T, Maskey-Warzechowska M, Grubek-Jaworska H, Chazan R (2012) Bronchoalveolar lavage total cell count in interstitial lung diseases_does it matter? Inflammation 35:803-809

Fang X, Bai C, Wang X (2012) Bioinformatics insights into acute lung injury/ acute respiratory distress syndrome. Clin Transl Med 2012:1

Faridvand Y, Nozari S, Asoudeh-Fard A, Karimi MA, Pezeshkian M, Safaie N, Nouri M (2017) Bovine lactoferrin ameliorates antioxidant esterase activity and 8-isoprostane levels in high-cholesterol-diet fed rats. Int Vitam Nutr Res 87(3-4):201-206

Favarin DC, de Oliveira JR, de Oliveira CJ, Rogerio Ade P (2013) Potential effects of medicinal plants and secondary metabolites on acute lung injury. Biomed Res Int 2013:576479

Filgueiras LR Jr, Martins JO, Serezani CH, Capelozzi VL, Montes MB, Jancar S (2012) Sepsis-induced acute lung injury (ALI) is milder in diabetic rats and correlates with impaired NFkB activation. PLoS ONE 7(9):e44987

Fujishima S (2016) Organ dysfunction as a new standard for defining sepsis. Inflamm Regen 36:24

Hegazy R, Salama A, Mansour D, Hassan A (2016) Renoprotective effect of lactoferrin against chromium-induced acute kidney injury in rats: involvement of IL-18 and IGF-1 inhibition. PLOS ONE 11(3):e0151486

Huang C, Pan L, Lin F, Dai H, Fu R (2017) Monoclonal antibody against toll-like receptor 4 attenuates ventilator-induced lung injury in rats by inhibiting MyD88- and NF-KB-dependent signaling. Int J Mol Med 39:693-700

Kellner M, Noonepalle S, Lu Q, Srivastava A, Zemskov E, Black SM (2017) ROS signaling in the pathogenesis of acute lung injury (ALI) and acute respiratory distress syndrome (ARDS). Adv Exp Med Biol 967:105-137

Kothari N, Bogra J, Abbas H, Kohli M, Malik A, Kothari D, Srivastava S, Singh PK (2013) Tumor necrosis factor gene polymorphism results in high TNF level in sepsis and septic shock. Cytokine 61(2):676-681

Kwiecien S, Jasnos K, Magierowski M, Sliwowski Z, Pajdo R, Brzozowski B, Mach T, Wojcik D, Brzozowski T (2014) Lipid peroxidation, reactive oxygen species and antioxidative factors in the pathogenesis of gastric mucosal lesions and mechanism of protection against oxidative stress-induced gastric injury. J Physiol Pharmacol 65(5):613-622

Manzoni P, Rinaldi M, Cattani S, Pugni L, Romeo MG, Messner H, Stolfi I, Decembrino L, Laforgia N, Vagnarelli F, Memo L, Bordignon L, Saia OS, Maule M, Gallo E, Mostert M, Magnani C, Quercia M, Bollani L, Pedicino R, Renzullo L, Betta P, Mosca F, Ferrari F, Magaldi R, Stronati M, Farina D (2009) Bovine lactoferrin supplementation for prevention of late-onset sepsis in very low-birth-weight neonates: a randomized trial. JAMA 302(13):1421-1428

Matthay MA, Ware LB, Zimmerman GA (2012) The acute respiratory distress syndrome. J Clin Invest 122:2731-2740

Matute-Bello G, Frevert CW, Martin TR (2008) Animal models of acute lung injury. Am J Physiol Lung Cell Mol Physiol 295:L379-L399

Queiroz-Junior CM, Pacheco CM, Fonseca AH, Klein A, Caliari MV, de Francischi JN (2009) Myeloperoxidase content is a marker of systemic inflammation in a chronic condition: the example given by the periodontal disease in rats. Mediators Inflamm 2009:760837

Randhawa R, Bellingan G (2007) Acute lung injury. Anaesthesia Intensive Care Med 8:477-480

Taşcı Hí, Erikoğlu M, Toy H, Karaibrahimoğlu A (2017) Course of sepsis in rats with thyroid dysfunction. Turk J Surg 33(3):175-179

Toufekoula C, Papadakis V, Tsaganos T (2013) Compartmentalization of lipid peroxidation in sepsis by multidrug-resistant gram-negative bacteria: experimental and clinical evidence. Crit Care 17(1):R6

Valenti P, Frioni A, Rossi A, Ranucci S, De Fino I, Cutone A, Rosa L, Bragonzi A, Berlutti F (2017) Aerosolized bovine lactoferrin reduces neutrophils and pro-inflammatory cytokines in mouse models of Pseudomonas aeruginosa lung infections. Biochem Cell Biol 95(1):41-47

Walkey AJ, Summer R, Ho V, Alkana P (2012) Acute respiratory distress syndrome: epidemiology and management approaches. J Clin Epidemiol 4:159-169

Ware LB, Matthay MA (2000) The acute respiratory distress syndrome. N Engl J Med 342:1334

Yalamati P, Bhongir AV, Karra M, Beedu SR (2015) Comparative analysis of urinary total proteins by bicinchoninic acid and pyrogallol red molybdate methods. J Clin Diagn Res 9:BC01-BC04

Zhang C, He Y, Shen Y (2019) L-Lysine protects against sepsis-induced chronic lung injury in male albino rats. Biomed Pharmacother 117:109043

\section{Publisher's Note}

Springer Nature remains neutral with regard to jurisdictional claims in published maps and institutional affiliations. 